\title{
Método de Chebyshev em Sistemas Não Lineares Singulares
}

\author{
Roberto Andreani, Sandra A. Santos, \\ Depto de Mat. Aplicada, IMECC, UNICAMP, \\ 15054-000, Campinas, SP \\ E-mail: andreani@ime.unicamp.br, sandra@ime.unicamp.br, \\ Wesley V. I. Shirabayashi Emerson V. Castelani, \\ Depto de Matemática - UEM \\ 88040-900, Maringá, PR \\ E-mail: wvishirabayashi@uem.br, evcastelani@uem.br.
}

Resumo: Vários autores mostraram que, sob certas condições, o método de Newton gera uma sequência linearmente convergente para uma solução quando a matriz Jacobiana é singular nesta solução. Neste trabalho temos por objetivo estender um resultado de convergência do método de Newton em sistemas não lineares singulares, para o método de Chebyshev. Através da análise de alguns exemplos conjecturamos a razão de convergência para o método de Chebyshev em sistemas singulares

Palavras-chave: Método de Newton, Método de Chebyshev, Sistemas não lineares singulares

\section{Exposição do Problema}

Considere um sistema não linear da forma:

$$
F(x)=0,
$$

onde $F: \mathbb{R}^{n} \longrightarrow \mathbb{R}^{n}$.

Existem vários trabalhos sobre a convergência do método de Newton quando a solução do problema (1) é um ponto de singularidade da matriz Jacobiana de $F, F^{\prime}(x)$, por exemplo $[2,3,4,5]$. Nos baseamos nos resultados de [2] para obter um resultado de convergência para o método de Chebyshev. Para mais detalhes sobre o método de Chebyshev ver $[1,6,7]$.

Para prosseguir a análise vejamos algumas notações: Seja $x_{*}$ uma solução de (1) tal que $F^{\prime}\left(x_{*}\right)$ seja singular e suponha que $F^{\prime}(x)$ seja inversível em

$$
\mathcal{V}_{\delta}=\left\{x \mid 0<\left\|x-x_{*}\right\|<\delta\right\}
$$

para algum $\delta>0$. Sejam também $N$ o núcleo de $F^{\prime}\left(x_{*}\right), X$ tal que $\mathbb{R}^{n}=N \oplus X, P_{N}$ o projetor em $N$ paralelo a $X$ e $P_{X}=I-P_{N}$ o projetor em $X$ paralelo a $N$.

Observação 1. Note que $X$ não é necessariamente o complemento ortogonal de $N$, apenas um subespaço que completa $N$ como soma direta para o $\mathbb{R}^{n}$.

Vamos utilizar as seguintes hipóteses:

H1: A função $F \in C^{3}$;

H2: O sistema $F(x)=0$ tem uma solução $x_{*}$;

H3: Existe $\delta>0$ tal que $F^{\prime}(x)$ é não singular na vizinhança $\mathcal{V}_{\delta}$, definida em $(2)$;

H4: Existe uma constante $c$ positiva tal que para todo $s \in N$ e $x \in \mathbb{R}^{n}$ vale a desigualdade:

$$
\left\|F^{\prime \prime}\left(x_{*}\right) s x\right\| \geq c\|s\| \cdot\|x\| .
$$


H5: $F^{\prime}\left(x_{*}\right) X=X$, isto é, o subespaço $X$ é invariante pela ação do operador $F^{\prime}\left(x_{*}\right)$.

Notação: No decorrer do texto usaremos as seguintes notações:

$$
\begin{gathered}
F_{k}=F\left(x_{k}\right), \quad F_{k}^{\prime}=F^{\prime}\left(x_{k}\right), \quad F_{k}^{\prime \prime}=F^{\prime \prime}\left(x_{k}\right), \\
F_{*}=F\left(x_{*}\right), \quad F_{*}^{\prime}=F^{\prime}\left(x_{*}\right), \quad F_{*}^{\prime \prime}=F^{\prime \prime}\left(x^{*}\right) .
\end{gathered}
$$

\section{Formulação Utilizada}

O método de Newton para resolver (1) é definido iterativamente por:

$$
x_{k+1}=x_{k}+d_{N},
$$

onde $d_{N}$ é solução de:

$$
F_{k}^{\prime} d_{N}=-F_{k}
$$

De outro modo:

$$
x_{k+1}=G\left(x_{k}\right),
$$

onde

$$
G(x)=\left\{\begin{array}{cc}
x-\left[F^{\prime}(x)\right]^{-1} F(x), & \text { se } x \neq x_{*} \\
x_{*}, & \text { se } x=x_{*}
\end{array}\right.
$$

é chamada função de iteração do método de Newton. Observe que no caso singular a primeira expressão para $G$ não vale em $x_{*}$, por isso definimos $G\left(x_{*}\right)=x_{*}$, posteriormente mostraremos que desse modo a aplicação $G$ é contínua.

O seguinte resultado refere-se a propriedades da função $G$ e sua utilidade ficará clara mais a frente.

Proposição 2. Suponha que $F \in C^{2}$. Para todo $x \in \mathcal{V}_{\delta}$, a aplicação $G(x)$ definida em (3) é diferenciável e $G^{\prime}(x)=\left[F^{\prime}(x)\right]^{-1} F^{\prime \prime}(x)\left[F^{\prime}(x)\right]^{-1} F(x)$.

Demonstração: Para $x \in \mathcal{V}_{\delta} \operatorname{temos} G(x)=x-\left[F^{\prime}(x)\right]^{-1} F(x)$, então

$$
F^{\prime}(x) G(x)=F^{\prime}(x) x-F(x) .
$$

Derivando com relação a $x$, obtemos:

$$
F^{\prime \prime}(x) G(x)+F^{\prime}(x) G^{\prime}(x)=F^{\prime \prime}(x) x+F^{\prime}(x) I-F^{\prime}(x)=F^{\prime \prime}(x) x .
$$

Assim, substituindo a expressão (3) e simplificando vem:

$$
F^{\prime}(x) G^{\prime}(x)=F^{\prime \prime}(x) x-F^{\prime \prime}(x) G(x)=F^{\prime \prime}(x)\left[F^{\prime}(x)\right]^{-1} F(x) .
$$

Portanto,

$$
G^{\prime}(x)=\left[F^{\prime}(x)\right]^{-1} F^{\prime \prime}(x)\left[F^{\prime}(x)\right]^{-1} F(x) .
$$

O resultado a seguir, sobre a invariância dos iterandos do método de Newton por transformações não singulares, é bem conhecido e de simples demonstração, mas damos ênfase a ele pois ele também é válido para o método de Chebyshev.

Lema 3. Os iterandos gerados pelo método de Newton são invariantes por transformações não singulares.

O teorema de convergência será precedido por alguns lemas técnicos, que foram extraídos do desenvolvimento da prova de Cavanagh para simplificar a apresentação dos resultados.

O lema a seguir serve como base para a demonstração de um outro lema, enunciado posteriormente, o qual estabelece que mesmo $F^{\prime}\left(x^{*}\right)$ sendo singular é possível obter um limitante para $\left[F^{\prime}(x)\right]^{-1}$ quando $x$ está próximo de $x^{*}$. Estes lemas também foram utilizados por Reddien [8]. 
Lema 4. Suponha a validade de H1, H2, H3, H4 e H5. Então

i) Existem $c_{1}>0$ e $\delta>0$ tais que $\left\|F^{\prime}(x) y\right\| \geq c_{1}\|y\|$ para todo $y \in X$ e $x \in \mathcal{V}_{\delta}$.

ii) Existem $c_{2}>0$ e $\delta>0$ tais que $\left\|F^{\prime}(x) s\right\| \geq c_{2}\left\|x-x_{*}\right\|\|s\|$ para todo $s \in N$ e $x \in \mathcal{V}_{\delta}$.

Lema 5. Suponha a validade de H1, H2, H3, H4 e H5. Então, existem $c_{3}>0$ e $\delta>0$ tais que para todo $x \in \mathcal{V}_{\delta}$

$$
\left\|\left(F^{\prime}(x)\right)^{-1}\right\| \leq \frac{1}{c_{3}\left\|x-x_{*}\right\|} .
$$

O teorema a seguir é o resultado de Cavanagh [2, Teorema 2.7] com leves modificações. Essas modificações foram feitas apenas para simplificar a demonstração. Também vale observar que a correção sugerida por Cavanagh para restaurar a convergência quadrática é apenas teórica e não pode ser utilizada na prática, pois necessita de prévio conhecimento da solução.

Teorema 6. Suponha a validade de H1, H2, H3, H4 e H5, e escolha um $\delta>0$ de modo que os Lemas 4 e 5 sejam válidos.

Então, $x_{*}$ é um ponto atrator para o método de Newton, a convergência é linear,

$$
P_{X}\left(x_{k}-x_{*}\right) \longrightarrow 0 \text { superlinearmente, } \frac{\left\|P_{N}\left(x_{k}-x_{*}\right)\right\|}{\left\|P_{N}\left(x_{k-1}-x_{*}\right)\right\|} \longrightarrow \frac{1}{2} \text { e } \frac{1}{2} P_{N}=G^{\prime}\left(x_{*}\right) .
$$

A convergência quadrática pode ser restaurada usando o seguinte processo iterativo:

$$
x_{k+1}=x_{k}+d_{N}-\frac{1}{2} P_{N}\left(x_{k}-x_{*}\right) .
$$

O seguintes resultados são consequências diretas do Teorema 6:

Corolário 7. A aplicação $G$ como definida em (3) é contínua em $\mathcal{V}_{\delta} \cup\left\{x_{*}\right\}$.

Corolário 8. O operador $G^{\prime}(x)$ definido por:

$$
G^{\prime}(x)=\left\{\begin{array}{cc}
{\left[F^{\prime}(x)\right]^{-1} F^{\prime \prime}(x)\left[F^{\prime}(x)\right]^{-1} F(x),} & \text { se } x \neq x_{*} \\
\frac{1}{2} P_{N}, & \text { se } x=x_{*}
\end{array}\right.
$$

é contínuo em $\mathcal{V}_{\delta} \cup\left\{x_{*}\right\}$.

\section{Resultados sobre o Método de Chebyshev}

O método de Chebyshev é um dos mais conhecidos métodos tensoriais para se resolver sistemas não lineares. Gundersen e Steihaug, em [6], mostraram que este método pode ser definido do seguinte modo:

$$
x_{k+1}=x_{k}+d_{N}+d_{C},
$$

no qual $d_{N}$ e $d_{C}$ são soluções dos seguintes sistemas lineares:

$$
F_{k}^{\prime} d_{N}=-F_{k}, \quad F_{k}^{\prime} d_{C}=-\frac{1}{2} F_{k}^{\prime \prime} d_{N} d_{N}
$$

Mostraremos que o Teorema 6 vale também para (5)-(6), ou seja, o método de Chebyshev gera uma sequência convergente para uma solução do problema (1). A ordem de convergência também é linear, mas o passo corretor, $d_{C}$, nos dá uma melhora com relação ao método de Newton. Mostraremos que a direção corretora, $d_{C}$, tem uma estreita relação com a correção sugerida por Cavanagh em [2]. As hipóteses são as mesmas do Teorema 6.

Teorema 9. Suponha a validade das hipóteses H1, H2, H3, H4 e H5 para $F: \mathbb{R}^{n} \longrightarrow \mathbb{R}^{n}$. Então, uma sequência gerada pelo método de Chebyshev converge para $x_{*} e$ $d_{C} \longrightarrow \frac{1}{4} P_{N}\left(x_{k}-x_{*}\right)$. 
Demonstração: Da relação (5) e da expansão de Taylor de segunda ordem para $F$ em torno de $x_{k} \in \mathcal{V}_{\delta}$ vem:

$$
\begin{aligned}
x_{k+1}-x_{*} & =x_{k}-x_{*}-\left(F_{k}^{\prime}\right)^{-1} F_{k}-\frac{1}{2}\left(F_{k}^{\prime}\right)^{-1} F_{k}^{\prime \prime} d_{N} d_{N} \\
& =\left(F_{k}^{\prime}\right)^{-1}\left[-F_{k}+F_{k}^{\prime}\left(x_{k}-x_{*}\right)-\frac{1}{2} F_{k}^{\prime \prime} d_{N} d_{N}\right] \\
& =\left(F_{k}^{\prime}\right)^{-1}\left[\frac{1}{2} F_{k}^{\prime \prime}\left(x_{k}-x_{*}\right)\left(x_{k}-x_{*}\right)+O\left(\left\|x_{k}-x_{*}\right\|^{3}\right)-\frac{1}{2} F_{k}^{\prime \prime} d_{N} d_{N}\right] \\
& =\left(F_{k}^{\prime}\right)^{-1}\left[\frac{1}{2} F_{k}^{\prime \prime}\left(x_{k}-x_{*}+d_{N}\right)\left(x_{k}-x_{*}-d_{N}\right)+O\left(\left\|x_{k}-x_{*}\right\|^{3}\right)\right] .
\end{aligned}
$$

Vejamos estimativas para os termos que aparecem na equação (7):

- $x_{k}-x_{*}+d_{N}=O\left(\left\|x_{k}-x_{*}\right\|\right)$

De fato, pela expansão de Taylor temos:

$$
F_{*}=F_{k}+F_{k}^{\prime}\left(x_{*}-x_{k}\right)+O\left(\left\|x_{k}-x_{*}\right\|^{2}\right) .
$$

Como $F_{*}=0$, segue que:

$$
-F_{k}+F_{k}^{\prime}\left(x_{k}-x_{*}\right)=O\left(\left\|x_{k}-x_{*}\right\|^{2}\right)
$$

Assim, do Lema 5 vem:

$$
\begin{aligned}
x_{k}-x_{*}+d_{N} & =x_{k}-x_{*}-\left(F_{k}^{\prime}\right)^{-1} F_{k} \\
& =\left(F_{k}^{\prime}\right)^{-1}\left[-F_{k}+F_{k}^{\prime}\left(x_{k}-x_{*}\right)\right] \\
& =\left(F_{k}^{\prime}\right)^{-1} O\left(\left\|x_{k}-x_{*}\right\|^{2}\right) \\
& =O\left(\left\|x_{k}-x_{*}\right\|\right) .
\end{aligned}
$$

- $x_{k}-x_{*}-d_{N}=O\left(\left\|x_{k}-x_{*}\right\|\right)$

Primeiro note que:

$$
\begin{aligned}
\left(F_{k}^{\prime}\right)^{-1} F_{k} & =\left(F_{k}^{\prime}\right)^{-1}\left[F_{*}^{\prime}\left(x_{k}-x_{*}\right)+O\left(\left\|x_{k}-x_{*}\right\|^{2}\right)\right] \\
& \left.=\left(F_{k}^{\prime}\right)^{-1} F_{*}^{\prime}\left(P_{X}+P_{N}\right)\left(x_{k}-x_{*}\right)+\left(F_{k}^{\prime}\right)^{-1} O\left(\left\|x-x_{*}\right\|^{2}\right)\right] \\
& \left.=\left(F_{k}^{\prime}\right)^{-1} F_{*}^{\prime} P_{X}\left(x_{k}-x_{*}\right)+\left(F_{k}^{\prime}\right)^{-1} O\left(\left\|x-x_{*}\right\|^{2}\right)\right] .
\end{aligned}
$$

Então, aplicando o Lema 5, obtemos:

$$
\left\|d_{N}\right\|=\left\|\left(F_{k}^{\prime}\right)^{-1} F_{k}\right\| \leq c_{6}\left\|x_{k}-x_{*}\right\|,
$$

para $x_{k} \in \mathcal{V}_{\delta}$ e $c_{6}$ uma constante positiva.

Assim,

$$
\left\|x_{k}-x_{*}-d_{N}\right\| \leq\left\|x_{k}-x_{*}\right\|+\left\|d_{N}\right\| \leq c_{7}\left\|x_{k}-x_{*}\right\| .
$$

Ou seja,

$$
x_{k}-x_{*}-d_{N}=O\left(\left\|x_{k}-x_{*}\right\|\right) \text {. }
$$

Retornando a (7),

$$
\begin{aligned}
x_{k+1}-x_{*} & =\frac{1}{2}\left(F_{k}^{\prime}\right)^{-1} F_{k}^{\prime \prime} O\left(\left\|x_{k}-x_{*}\right\|\right) O\left(\left\|x_{k}-x_{*}\right\|\right)+\left(F_{k}^{\prime}\right)^{-1} O\left(\left\|x_{k}-x_{*}\right\|^{3}\right) \\
& =O\left(\left\|x_{k}-x_{*}\right\|\right)+O\left(\left\|x_{k}-x_{*}\right\|^{2}\right)=O\left(\left\|x_{k}-x_{*}\right\|\right) .
\end{aligned}
$$


A última afirmação do enunciado segue de observar que,

$$
d_{C}=-\frac{1}{2}\left(F_{k}^{\prime}\right)^{-1} F_{k}^{\prime \prime} d_{N} d_{N}=\frac{1}{2} G_{k}^{\prime} d_{N} .
$$

De certo modo, a direção $d_{C}$ aproxima a correção teórica sugerida por Cavanagh $\left[\frac{1}{2} P_{N}\left(x_{k}-x_{*}\right)\right]$. Nos seguintes exemplos unidimensionais vemos a melhora obtida pelo método de Chebyshev em comparação com Newton.

Exemplo 10. Considere $F(x)=x^{2}, x \in \mathbb{R}$. Vejamos como se comportam as sequências geradas pelos métodos de Newton e Chebyshev para resolver $F(x)=0$.

Temos, $F^{\prime}(x)=2 x$ e $F^{\prime \prime}(x)=2$. Todas as hipóteses dos Teoremas 6 e 9 são satisfeitas.

- Newton

$$
x_{k+1}=\frac{1}{2} x_{k}, \text { ou seja, } \frac{x_{k+1}}{x_{k}}=\frac{1}{2} .
$$

- Chebyshev

$$
x_{k+1}=\frac{3}{8} x_{k}, \text { ou seja, } \frac{x_{k+1}}{x_{k}}=\frac{3}{8} .
$$

Exemplo 11. Considere agora $F(x)=x^{3}+c x^{2}, x \in \mathbb{R}$ e c uma constante real não nula. Façamos a mesma análise do exemplo anterior:

Temos, $F^{\prime}(x)=3 x^{2}+2 c x$ e $F^{\prime \prime}(x)=6 x+2 c$. Neste caso a equação $F(x)=0$ tem duas raízes, a saber, $x_{*}=-c$ e $x_{*}=0$, no entanto estamos interessados no caso em que $F^{\prime}(x)=0$, ou seja, $x_{*}=0$. As hipóteses dos Teoremas 6 e 9 são satisfeitas.

- $\underline{\text { Newton }}$

$$
x_{k+1}=x_{k}-\frac{x_{k}^{3}+c x_{k}^{2}}{3 x_{k}^{2}+2 c x_{k}} .
$$

E temos que:

$$
\frac{x_{k+1}}{x_{k}} \longrightarrow \frac{1}{2} \text { quando } x_{k} \longrightarrow x_{*}
$$

- Chebyshev

$$
x_{k+1}=x_{k}-\frac{x_{k}^{3}+c x_{k}^{2}}{3 x_{k}^{2}+2 c x_{k}}-\frac{x_{k}\left(3 x_{k}+c\right)\left(x_{k}+c\right)^{2}}{\left(3 x_{k}+2 c\right)^{3}} .
$$

Neste caso temos:

$$
\frac{x_{k+1}}{x_{k}} \longrightarrow \frac{3}{8} \text { quando } x_{k} \longrightarrow x_{*}
$$

Comentário 12. Estes exemplos nos levam a conjecturar que a razão de convergência do método de Chebyshev é $\frac{3}{8}$.

Uma modificação que fizemos no método de Chebyshev para melhorar a correção no sentido sugerido por Cavanagh foi adotar a sobrerelaxação $d_{C m}=2 d_{C}$, o que chamamos de Chebyshev modificado. Neste caso, o processo iterativo torna-se:

$$
x_{k+1}=x_{k}+d_{N}+2 d_{C} .
$$

Assim temos $d_{C m} \longrightarrow \frac{1}{2} P_{N_{1}}\left(x_{k}-x_{*}\right)$, e fazendo a mesma análise do Exemplo 10 obtemos:

$$
x_{k+1}=\frac{1}{4} x_{k} \quad \text { e } \frac{x_{k+1}}{x_{k}}=\frac{1}{4} .
$$

Já para o Exemplo 11 obtemos:

$$
x_{k+1}=x_{k}-\frac{x_{k}^{3}+c x_{k}^{2}}{3 x_{k}^{2}+2 c x_{k}}-\frac{2 x_{k}\left(3 x_{k}+c\right)\left(x_{k}+c\right)^{2}}{\left(3 x_{k}+2 c\right)^{3}}
$$




$$
\frac{x_{k+1}}{x_{k}} \longrightarrow \frac{1}{4} \text { quando } x_{k} \longrightarrow x_{*} \text {. }
$$

O próximo resultado é uma adaptação do Teorema 9 para o método definido por (8).

Teorema 13. Suponha as mesmas hipóteses do Teorema 9. Então, se $x_{0}$ é tomado suficientemente próximo a $x_{*}$, o método definido por (8) gera uma sequência convergente a $x_{*}$.

Demonstração: Utilizando a mesma técnica da prova do Teorema 9, segue de (8) que:

$$
\begin{aligned}
x_{k+1}-x_{*} & =x_{k}-x_{*}-\left(F_{k}^{\prime}\right)^{-1} F_{k}-\left(F_{k}^{\prime}\right)^{-1} F_{k}^{\prime \prime} d_{N} d_{N} \\
& =\left(F_{k}^{\prime}\right)^{-1}\left[-F_{k}+F_{k}^{\prime}\left(x_{k}-x_{*}\right)\right]-\left(F_{k}^{\prime}\right)^{-1} F_{k}^{\prime \prime} d_{N} d_{N} .
\end{aligned}
$$

Agora usando o Lema 5 e os seguintes fatos, já mostrados:

$$
-F_{k}+F_{k}^{\prime}\left(x_{k}-x_{*}\right)=O\left(\left\|x_{k}-x_{*}\right\|^{2}\right), \quad d_{N}=O\left(\left\|x_{k}-x_{*}\right\|\right),
$$

obtemos,

$$
x_{k+1}-x_{*}=O\left(\left\|x_{k}-x_{*}\right\|\right) .
$$

\section{Conclusões}

Neste trabalho apresentamos um resultado de convergência local do método de Newton em sistemas singulares, originalmente feito por Cavanagh em [2], e estendemos tal resultado para o método de Chebyshev. Também modificamos os iterandos do método de Chebyshev obtendo um novo método, chamado por nós de método de Chebyshev modificado. Pela análise de alguns exemplos conjecturamos que o método de Chebyshev tem razão de convergência $\frac{3}{8}$ e o método de Chebyshev modificado tem razão de convergência $\frac{1}{4}$. Uma possibilidade de trabalho futuro é tentar mostrar que essa conjectura é verdadeira.

\section{Referências}

[1] V. Candela, A. Marquina, Recurrence relations for rational cubic methods II: the Chebyshev method. Computing, 45 (1990) 355-367.

[2] R.C. Cavanagh, "Difference equations and iterative process", Thesis, Computer Sci. Dept., Univ. of Maryland, College Park, 1970.

[3] D.W. Decker, H.B. Keller, C.T. Kelley, Convergence rates for Newton's method at singular points. SIAM J. of Numer. Anal., 20 (1983) 296-314.

[4] D.W. Decker, C.T. Kelley, Newton's method at singular points, I. SIAM J. of Numer. Anal., 17 (1980) 66-70.

[5] A. Griewank, M.R. Osborne, Newton's method for singular problems when the dimension of the null space is > 1. SIAM J. of Numer. Anal., 18 (1981) 145-149.

[6] G. Gundersen, T. Steihaug, On large scale unconstrained optimization problems and higher order methods. Opt. Methods and Software, 25 (2010) 337-358.

[7] M.A. Hernández, M.A. Salanova, A family of Chebyshev-Halley type methods. Intern. J. of Computer Math., 47 (1993) 59-63.

[8] G.W. Reddien, On Newton's method for singular problems. SIAM J. of Numer. Anal., 15 (1978) 993-996. 